\title{
Posture and Vibration Control Based on Virtual Suspension Model for Multi-Legged Walking Robot
}

\author{
Qingjiu Huang \\ Tokyo Institute of Technoligy \\ Japan
}

\section{Introduction}

Until now, studies on suspension control by using frequency response analysis that have been reported are almost for motorcar, but not for multi-legged walking robot. However, because of the disturbances, such as the various frequency properties of terrain, the collision and the slip between foot of robot and ground, the dynamic changes of the supported weight by each leg and the centre of gravity of robot with the change of the walking pattern, tiny vibration of robot's body occur when robot walks, especially on rough terrain. This tiny vibration can become unstable big vibration when the above disturbances to the posture of robot exist, which will influence normal walk and work of robot. Recently there are some studies about the posture control of multi-legged walking robot (Shin-Min Song \& Keneth J.Waldron, 1989); (Kan Yoneda, et al, 1994); (Qingiu Huang, et al, 2000); (Qingjiu Huang \& Kenzo Nonami, 2002); (Qingjiu Huang, et al, 2003), but no any study on the tiny vibration ठ․ control of robot's body. Moreover, only posture control can not effectively decrease the tiny ¿ vibration of body when robot walks, especially on rough terrain. Therefore, it is necessary to study suspension and its control algorithm to decrease the tiny vibration of body for multi을 legged walking robot. In this chapter, we treat a six-legged walking robot as a study 을 example of the multi-legged walking robot, and introduce the newest study on a control for $\stackrel{\Phi}{.1}$ the posture and vibration of the robot using suspension mechanism to realize the better stability and the better adaptability of its walking for unknown rough terrain.

On the other hand, until now most reported studies on suspension control are performed on $\$$ the basis of real suspension model with spring and damper (Nurkan Yagiz, et al, 2000);

đ్ (Makoto Yokoyama, et al, 2001). However, for large scale multi-DOF system, such as multi-

T] legged walking robots, it is difficult to equip lots of springs and dampers to the robot.

तٓ Therefore, in this chapter, we introduce a new control method for the posture and vibration

of the six-legged walking robot (Qingjiu Huang, et al, 2007), which is based on not a real

o suspension model but a virtual suspension model consisting of virtual spring and damper.

And then considering the nonlinear disturbances and trade-off problem in the design of

$ᄃ$ suspension, a robust control using sliding mode control based on the constructed virtual ญे suspension model for the posture and vibration of the six-legged walking robot is proposed and introduced.

Source: Bioinspiration and Robotics: Walking and Climbing Robots, Book edited by: Maki K. Habib ISBN 978-3-902613-15-8, pp. 544, I-Tech, Vienna, Austria, EU, September 2007 
The chapter is organized as follows. In section 2, by the introduction of developing a sixlegged walking robot for this study based on stable theory of wave gaits and CAD dynamic model(Qingjiu Huang, et al, 2004), we offered a more efficiency and more effective developing technique for a large scale multi-DOF dynamic system, such as multi-legged walking robot. Then in section 3, we introduce the design of a virtual suspension model with one degree of freedom, which has virtual spring and damper, for the direction of the centre of gravity, the pitch angle, and the roll angle of body respectively, to keep the posture stability of body when robot walks. In section 4 , in order to decrease the vibration of body when robot walks, an active suspension control by using sliding mode control based on a virtual suspension model is designed by using two kinds of sliding mode control, the one of servo style and the one based on mode coordinate are designed. In section 5 , the above posture and vibration control methods are discussed using the walking experimental results of the developed six-legged walking robot. Finally, the conclusions will be presented in section 6 .

\section{Development of a Six-Legged Walking Robot Based on Stable Theory of Wave Gaits and CAD Dynamic Model}

In this section, we introduce a new effective technique for developing a multi-legged walking robot. In this technique, the size of robot body is designed by using the constrained condition of the stability margin theorem in wave gaits (Shin-Min Song \& Keneth J.Waldron, 1989). And the motors attached to each leg are selected depending on the analysis result of the dynamic characteristics of robot by using a CAD dynamic model. By this technique, more efficiency and more effective developing method of robot was offered, and the robot with more stable mobility was developed. As a result, our developed robot with three degrees of freedom in each leg, like a crab, can move in all directions, and it's leg ahead can stop freely in the arbitrary position of the three-dimensional space.

\subsection{Design of the Size of Robot}

The size of the robot body was designed using the constrained condition of the stability margin theorem in wave gaits. Wave gait is a kind of gait that on the instance of one leg touchdown one of the fore legs is raised, and the up-and-down action is transported from the hind leg to the fore leg like a wave. One important variable for deciding the size of the robot body, $P$, is obtained according to the stability margin $S>0$ and desired step distance R. $S$ which is defined in Ref.(1) is given by the following equations for a 2 -legged walking robot.

In the case of $1 / 2 \leq \beta<2 / 3$ and $R i \leq R b i$

$$
S_{i}=(n / 2-1) P i+(1-3 /(4 \beta)) R_{i} \quad(i=x, y)
$$

In the case of $\beta>2 / 3$ and $R i>R b i$

$$
S_{i}=(n / 2-1) P i+(1 /(4 \beta)-1 / 2) R i
$$

where, $R b i$ is obtained by using

$$
R b i=|\beta /(3 \beta-2)| P_{i}
$$


$P$ is the stroke pitch denoting the distance between the centres of strokes of the adjacent legs on one side. $R$ is the leg stroke denoting the distance through which the foot is translated relative to the body during the support phase. $\beta$ is the duty factor denoting the time fraction of a cycle time in which a leg is in the support phase.

Here, the stroke pitches between two legs shown in Fig.1 in $x$ direction $P x$ and $y$ direction $P y$ were designed based on the tripod gait. In the case of tripod gait, $\beta=1 / 2$. And, in this research, in order to realize stable walking, we set the target values of the stability margin as $S_{x}=0.30 \mathrm{~m}, S_{y}=0.025 \mathrm{~m}$, of the walk space as $R x=0.15 \mathrm{~m}, R y=0.20 \mathrm{~m}$. Substituting these values into Eq.(1) obtained the size of robot body, that is $P x=0.75 \mathrm{~m}, P y=0.25 \mathrm{~m}$.

The sizes of the three links of the leg were obtained by using inverse kinematics in consideration of $20 \mathrm{~cm}$ high obstacles, so that the robot could climb ordinary stairs. Fig.1 shows the designed sizes of three links of each leg.

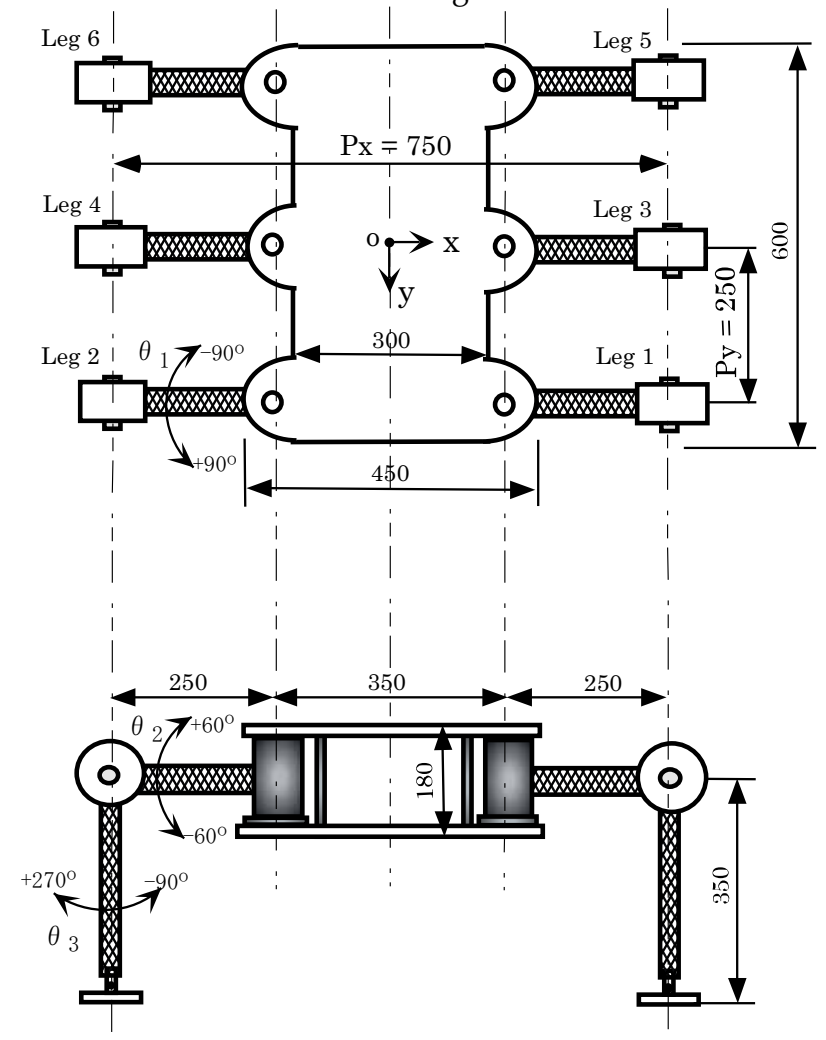

Figure 1. The size of our six-legged walking robot (Huang, Q. et al, 2007)

\subsection{Selection of the Motor of Joints}

Until now, in the conventional studies on the development of the walking robot, the selection of the motor of joint depends on the local dynamic analysis about one leg unit. However, it is not enough with the local dynamic analysis only for one leg unit because when the robot walks, not only one leg unit but also the centre of gravity of its body changes 
dynamically. Therefore, in this study, we selected the motor by the global analysis result over the body of robot by using a 3D CAD dynamic model.

Fig.2 shows a dynamic model for a six-legged walking robot with three joints in one leg. This dynamic model was built on the mechanism analysis software DADS (LMS DADS). The size of the model is the same as Fig.1. The mass, the moment of inertia, the constrained element, the friction, and the opposite force were set precisely, so it can be said that it is a dynamic model with high accuracy. Besides, since the action of this model is visible, it is convenient to check the actions of the simulations of various gaits.

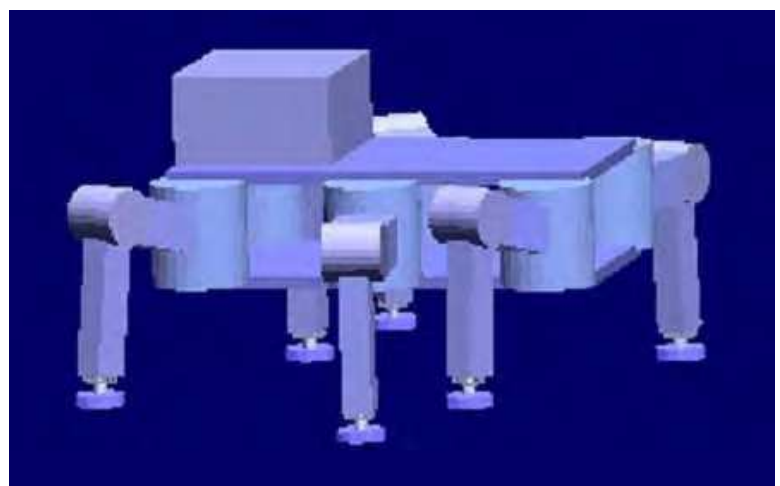

Figure 2. 3D CAD dynamic model for our six-legged walking robot (Huang, Q. et al, 2007)

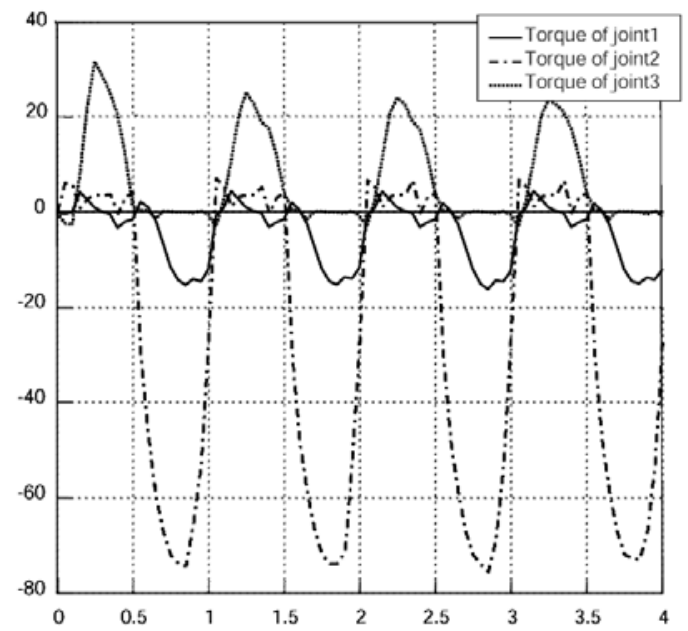

Figure 3. Torques of motors for tripod gait (Huang, Q. et al, 2007)

The simulation of the CAD model was performed with a tripod gait, which is a kind of gait that three legs move simultaneously when a six-legged walking robot is advancing. The tripod gait was used here since the load distribution on each joint of each leg is the highest. Fig.3 shows the torque curves of the 1st, 2nd, and 3rd joints of the leg, respectively, when the biggest torque happened. At this moment, the load on the robot was $40 \mathrm{~kg}$, the motion speed was $420 \mathrm{~m} / \mathrm{h}$, and the tripod gait was performed in 3 circles. The motor was selected according to the torque curves and the designed safety factor $a$. The running gear of each 
Posture and Vibration Control Based on Virtual Suspension Model for Multi-Legged Walking Robot

joint consisted of a DC motor and a harmonic driver with a deceleration ratio of 100 . The safety factor $a=1.2$ for the 1st and 2nd joints, and $a=1.5$ for the 3rd joint.

Each parameter encompassing the weight of each part of robot and the selected rated torque of the motor are shown in Table 1.

\begin{tabular}{|l|c}
\hline Weight of body frame [kg] & 15.0 \\
\hline Weight of one leg unit (include shoulder) $[\mathrm{kg}]$ & 6.0 \\
Total weight [kg] & 51.0 \\
Loadable weight [kg] & 40.0 \\
Reduction ratio of harmonic drive $\beta_{1}$ & 80 \\
Reduction ratio of harmonic drive $\beta_{2}$ & 120 \\
Reduction ratio of harmonic drive $\beta_{3}$ & 80 \\
Reduction ratio of timing belt $\gamma_{1}$ & 2.0 \\
Reduction ratio of timing belt $\gamma_{1}$ & 4.0 \\
Reduction ratio of timing belt $\gamma_{1}$ & 4.2 \\
Rated torque of DC motor $\tau_{r 1}[\mathrm{Nm}]$ & 0.14 \\
Rated torque of DC motor $\tau_{r 2}[\mathrm{Nm}]$ & 0.20 \\
Rated torque of DC motor $\tau_{r 3}[\mathrm{Nm}]$ & 0.14 \\
\hline
\end{tabular}

Table 1. Specifications of the Robot (Huang, Q. et al, 2007)

\subsubsection{The Developed Six-legged Walking Robot TRUTH}

Fig.4 shows the developed robot. The driving mechanism for each leg is a hybrid type mechanism composing a DC motor and a harmonic slowdown device through a rubber belt. Some accessories, such as computers, sensors, motor drive drivers and one AC power supply, are mounted on the body of robot. And we named the robot as TRUTH (Titech Robot walkable on Unstructure Terrain like Hexapod).

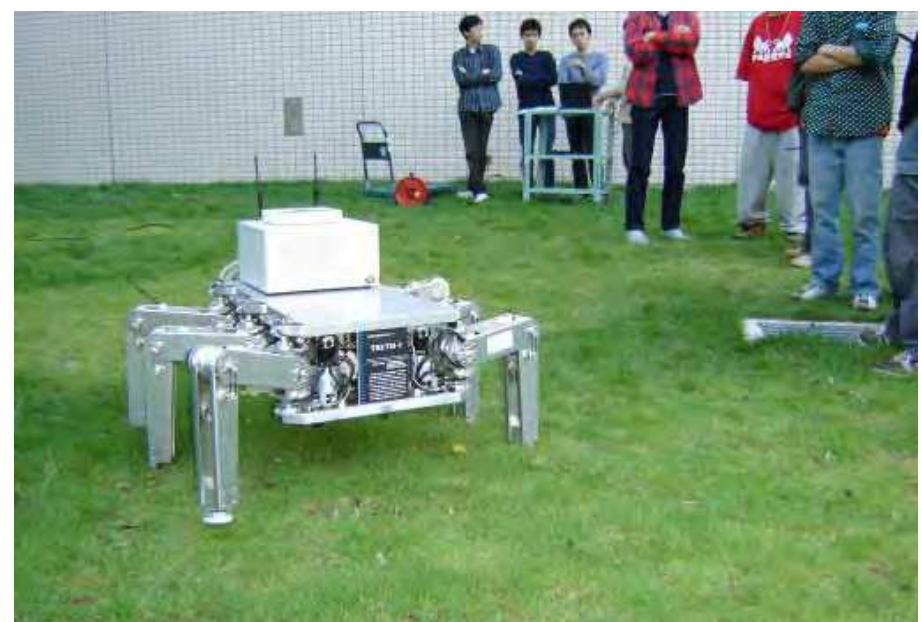

Figure 4. The developed six-legged walking robot (Huang, Q. et al, 2007) 


\section{Virtual Suspension Dynamic Model for the Multi-legged Robot}

As a generally known, the stability margin theorem which is statically analyzed from the viewpoint of geometry for the multi-legged robot posture, was studied well (Shin-Min Song \& Keneth J.Waldron, 1989). However, it is also necessary to analyze the dynamic characteristics of robot body in order to realize its stable posture. It is because when the robot walks the dynamic characteristics becomes one of the main elements which influence the posture of robot. Therefore, in this study, to analyze the dynamic characteristics of robot, the body of robot is assumed to be a solid of revolution, and each leg is assumed to be the support of the solid of revolution.

To restrain the vibration of robot body, a suspension model is built in this study. As shown in Fig.5, the ground is assumed to be rigid; a suspension model of one degree of freedom with virtual springs and dampers is designed in the vertical direction, the direction of the pitch angle, and the direction of the roll angle, respectively. In Fig.5, the vertical direction of the robot body is defined to be the $z$ coordinate axis, the advancing direction of the robot is defined to be the $y$ coordinate axis, and the direction crosshatched to the above two directions is defined to be the $x$ coordinate axis.

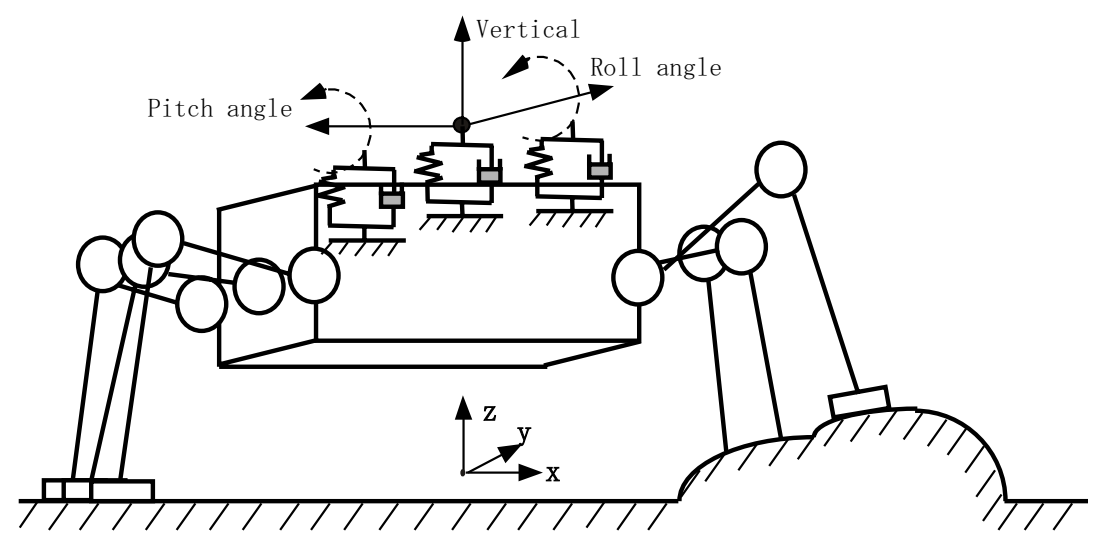

Figure 5. The virtual suspension dynamic model (Huang, Q. et al, 2007)

Here, $z, \theta_{p}, \theta_{r}$ are defined to be the changes in the vertical direction, the directions of the pitch angle and the roll angle from the balance place, respectively. When the robot is not walking, hence there are no disturbances, so the motion equation of the vibration system is a time invariable linear system as follows,

$$
\begin{gathered}
M \ddot{z}=-K_{z} z-C_{z} \dot{z} \\
I_{x} \ddot{\theta}_{p}=-K_{p} \theta_{p}-C_{p} \dot{\theta}_{p} \\
I_{y} \ddot{\theta}_{r}=-K_{r} \theta_{r}-C_{r} \dot{\theta}_{r}
\end{gathered}
$$

Where $M, I_{x}, I_{y}$ are the weight supported by support legs, the moment of inertia around $x$ axis coordinate, and the moment of inertia around $y$ axis coordinate respectively. $K_{z}$, 
$C_{z}, K_{p}, C_{p}, K_{r}, C_{r}$ are the coefficients of the springs and the dampers of the virtual dynamic model built in the vertical direction, the directions of the pitch angle and the roll angle, respectively. The characteristic equations of the three virtual dynamic models are expressed as

$$
\begin{gathered}
M s^{2}+C s+K=0 \\
s^{2}+2 \xi w_{n} s+w_{n}{ }^{2}=0
\end{gathered}
$$

where, $\boldsymbol{M}=M$ or $I_{x}$ or $I_{y}, \boldsymbol{C}=C_{z}$ or $C_{p}$ or $C_{r}, \boldsymbol{K}=K_{z}$ or $K_{p}$ or $K_{r}$.

The natural angular frequency of the virtual vibration system $w_{n}$ and the damping coefficient $\xi$ are,

$$
w_{n}=\sqrt{K / M}, \xi=C /\left(2 w_{n} M\right)
$$

In order to improve the excessive characteristic of the system response and to minify the overshot, the damping coefficient $\xi$ is selected to be within 0.7 $\square$ 1.0. Besides, the natural angular frequency $w_{n}$ is enlarged as much as possible to avoid resonance because the vibrations in the vertical direction, the directions of the pitch angle and the roll angle, the disturbances from collisions and slippage between the ground and the robot leg, and dynamic changes of the supported weight and the centre of gravity are within low frequency. Furthermore, because of the enlarging of the natural angular frequency $w_{n}$, good stability and excessive response characteristics within a wide frequency band can be realized in the virtual suspension dynamic system. Therefore, in this study, the natural angular frequency $w_{n}$ and the damping coefficient $\xi$ are selected respectively as follows,

$$
\begin{array}{lll}
\omega_{n z}=60 & \omega_{n p}=250 & \omega_{n r}=250 \\
\xi_{z}=0.800 & \xi_{p}=0.800 & \xi_{r}=0.800
\end{array}
$$

The body weight and rotary moment of inertia of the six-legged walking robot in this study are

$$
M=16.753[\mathrm{~kg}] \quad I_{x}=1.072\left[\mathrm{kgm}^{2}\right] \quad I_{y}=0.906\left[\mathrm{kgm}^{2}\right]
$$

From Eq.(9), the parameters of the proposed virtual dynamic model are as follows,

$$
\begin{array}{cc}
K_{z}=167530.0[\mathrm{~N} / \mathrm{m}] & C_{z}=2680.5[\mathrm{Ns} / \mathrm{m}] \\
K_{p}=67000.0[\mathrm{Nm} / \mathrm{rad}] & C_{p}=428.8[\mathrm{Nms} / \mathrm{rad}] \\
K_{r}=56643.8[\mathrm{Nm} / \mathrm{rad}] & C_{r}=428.8[\mathrm{Nms} / \mathrm{rad}]
\end{array}
$$

\section{Suspension Control Using Sliding Mode Control Based on the Virtual Dynamic Model}

4.1 Two Problems in the Design of Virtual Suspension for the Support Leg System

\subsubsection{Trade-off Problem in the Design of Suspension}

According to the virtual suspension model expressed by Equations (4), (5), and (6), we can determine the parameters $C$ and $K$, the spring coefficient and the damping coefficient of 
the suspension model shown as in section 3. However, In the case of multi-legged robot, because of the heavy weight of the robot, there is a trade-off problem in the design of suspension. This trade-off problem is, in order to cut the low frequency vibration $(3 \mathrm{~Hz}-$ $8 \mathrm{~Hz}$ ) from the walking pattern, the system is needed soft springs $K$ according to Eq.(9). But this means poor supporting force for the weight of the robot body, and also means poor ability of eliminating the stationary error of posture. Because of this trade-off problem, although only virtual suspension model can effectively decrease the vibrations of the robot body, it can not effectively realize the posture of the robot body.

\subsubsection{TIL Model Becomes TVN Model When Robot Walks}

When robot does not walk, the virtual dynamic model for robot posture is built as shown in Fig.5. This model is a TIL(Time Invariant Linear) model expressed by Equations (4), (5), and (6). However, the model for a walking robot becomes a TVN(Time Variant Nonlinear) model shown as the Equations (10), (11), and (12), when the robot walks because of dynamic changes in the COG, supported weight, and moment of inertia, and disturbances caused by collisions and slippage between the ground and the leg of the robot.

$$
\begin{gathered}
\left(M+\Delta M\left(t, \theta_{s w}\right)\right) \ddot{z}=-K_{z} z-C_{z} \dot{z}+\ddot{z}_{z} \\
\left(I_{x}+\Delta I_{x}\left(t, \theta_{s w}\right)\right) \ddot{\theta}_{p}=-K_{p} \theta_{p}-C_{p} \dot{\theta}_{p}+\ddot{z}_{p} \\
\left(I_{y}+\Delta I_{y}\left(t, \theta_{s w}\right)\right) \ddot{\theta}_{r}=-K_{r} \theta_{r}-C_{r} \dot{\theta}_{r}+\ddot{z}_{r}
\end{gathered}
$$

Where, $\boldsymbol{d}=\left[\begin{array}{lll}\ddot{z}_{z} & \ddot{z}_{p} & \ddot{z}_{r}\end{array}\right]^{T}$ are the disturbances items, and $\Delta M\left(t, \theta_{s w}\right), \quad \Delta I_{x}\left(t, \theta_{s w}\right)$, $\Delta I_{y}\left(t, \theta_{s w}\right)$ are the time variant items. $\theta_{s w}$ are the angles of each joint of the swing legs.

Therefore, a virtual dynamic model cannot eliminate the dynamic vibrations in the posture of a six-legged walking robot, which is a time variant nonlinear system. Furthermore, a virtual dynamic model cannot eliminate stationary position errors or stationary velocity errors.

\subsection{Solving the Above Two Problems Using Sliding Mode Control}

In order to solve the trade-off problem of the designing virtual suspension model, and deal with the time variant non-linear suspension system, we consider active control input $u_{z}, u_{p}$ and $u_{r}$ for the constructed virtual suspension model shown in Fig.6. Because the sliding mode control is very effective for time variant nonlinear systems and can eliminate stationary errors of position and velocity (Kenzo Nonami \& Hongqi Tian, 1994), in our study, the control inputs $u_{z}, u_{p}$ and $u_{r}$ were designed by using sliding mode control. Furthermore, the motion equations for the vertical direction, the directions of the pitch angle of robot body and the roll angle of robot body are defined as

$$
\begin{gathered}
\left(M+\Delta M\left(t, \theta_{s w}\right)\right) \ddot{z}=-K_{z} z-C_{z} \dot{z}+u_{z}+\ddot{z}_{z} \\
\left(I_{x}+\Delta I_{x}\left(t, \theta_{s w}\right)\right) \ddot{\theta}_{p}=-K_{p} \theta_{p}-C_{p} \dot{\theta}_{p}+u_{p}+\ddot{z}_{p} \\
\left(I_{y}+\Delta I_{y}\left(t, \theta_{s w}\right)\right) \ddot{\theta}_{r}=-K_{r} \theta_{r}-C_{r} \dot{\theta}_{r}+u_{r}+\ddot{z}_{r}
\end{gathered}
$$

In this study, we designed two type of sliding mode control, the one is a sliding mode control of servo system, and the other one is a sliding mode control based on the vibration mode coordinate. 


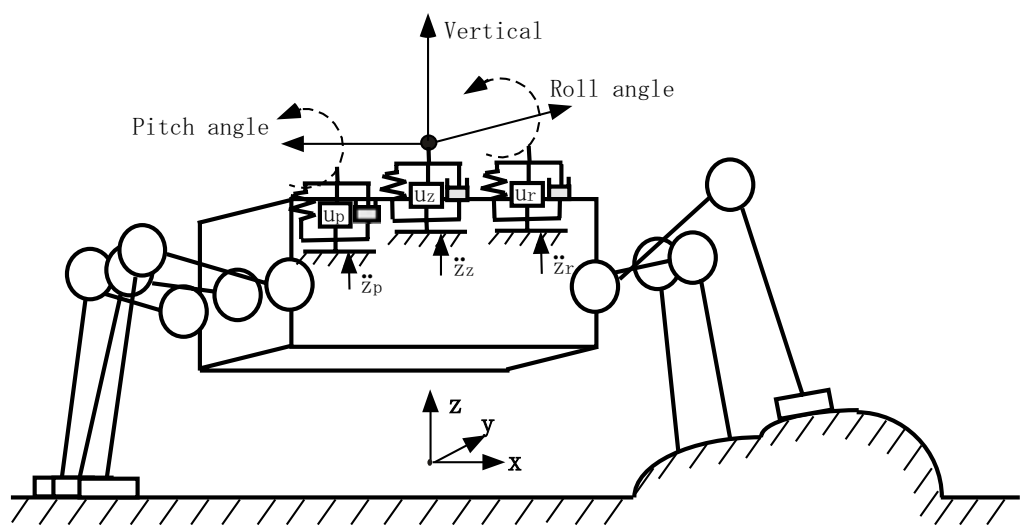

Figure 6 . The virtual suspension model considering of active control input and disturbances (Huang, Q. et al, 2007)

\subsection{A Sliding Mode Control of Servo System}

In this study, we firstly designed the same control system for the vertical direction, the direction of the pitch angle, and the direction of the roll angle. Here, only the designed sliding mode control of one-type style servo system in the direction of the pitch angle is introduced. In the designed sliding mode control, the integral value of the difference between the target pitch angle and the actual pitch angle is defined to be a new state variable. Substituting the new state variable $z_{\text {en }}$ into the Eq.(14), an extended state equation can be derived. The extended state equation and the switching function $\sigma$ are

$$
\left\{\begin{array}{c}
\dot{\boldsymbol{x}}_{e}=A \boldsymbol{x}_{e}+\boldsymbol{B} \boldsymbol{u}+\boldsymbol{Q} r+\boldsymbol{F} d \\
z_{e n}=\int\left(r-\theta_{p}\right) d t \quad \boldsymbol{x}_{e}=\left[\begin{array}{lll}
z_{e n} & \theta_{p} & \dot{\theta}_{p}
\end{array}\right]^{T} \quad d=\ddot{z}_{p} \\
\boldsymbol{s}=\boldsymbol{S} \boldsymbol{x}_{e n}
\end{array}\right.
$$

and, $A, B, Q$ are expressed by

$$
\boldsymbol{A}=\left[\begin{array}{ccc}
0 & -1 & 0 \\
0 & 0 & 1 \\
0 & -K_{p} / I_{p} & C_{p} / I_{p}
\end{array}\right] \quad \boldsymbol{B}=\left[\begin{array}{c}
0 \\
0 \\
1 / I_{p}
\end{array}\right] \quad \boldsymbol{Q}=\left[\begin{array}{l}
1 \\
0 \\
0
\end{array}\right]
$$

where, $r$ is the target value of the pitch angle, and is zero here. $d$ is the term denoting the disturbances. In sliding mode control, if the equivalent control input $u_{e q}$ is without consideration of disturbances, from $\sigma=\dot{\sigma}=0$, the linear input $u_{l p}$ can be obtained from

$$
u_{l p}=u_{e q}=-(S B)^{-1}\left(S A x_{e}+S Q r\right)
$$

Switching matrix $S$ is solved by using the solution of the Riccati equation. The real component of the eigenvalue of the equivalent control system is -50 here. The nonlinear input of the sliding mode control $u_{n l p}$ is expressed by

$$
u_{n l p}=-k(\boldsymbol{S B})^{-1} \frac{\sigma}{|\sigma|+\eta}
$$


Nonlinear input $u_{n l p}$ compensates for the uncertainty of the system, as the control input to constrain the system state variables within the switching plane. Here, the coefficient to repress the disturbances $k=8600$, and the coefficient to avoid the chattering $\eta=0.1$. Therefore, the input of the sliding mode control $u_{p}$ for the direction of pitch angle, which is composed of the linear control input $u_{l p}$ and the nonlinear control input $u_{n l p}$ is expressed as follows.

$$
u_{p}=u_{l p}+u_{n l p}=-(\boldsymbol{S B})^{-1}\left(\boldsymbol{S A} \boldsymbol{x}_{e}+S \boldsymbol{Q} r\right)-k(\boldsymbol{S B})^{-1} \frac{\sigma}{|\sigma|+\eta}
$$

The input of sliding mode control in the $z$ direction $u_{z}$ and the direction of roll angle $u_{r}$ were calculated in the same way by using Eqs.(16)-(19). Substituting the obtained $u_{z}, u_{p}$, and $u_{r}$ into Eqs.(13)-(15), the forces in $z$ direction of the tips of support legs were calculated according to (Kan Yoneda, et al, 1994). Then, by using position/force hybrid control introduced in (Qingjiu Huang \& Kenzo Nonami, 2002), the calculated forces of the tips of support legs were transformed into the motor torques to driving the motors attached on the support legs. On the other hand, the motor torques of the swing legs were calculated by PD control for following the desired trajectories of the swing legs.

\subsection{A Sliding Mode Control Based on the Vibration Mode Coordinate}

Also we designed a sliding mode control based on the vibration mode coordinate to control the posture and restrain the vibration of the robot body. Although the state variable is impossible to be controlled in the general state equation, by departing the mode, the state matrix in the state equation becomes a diagonal canonical matrix. Correspondingly, the state variable becomes controlled, and the system becomes easy to be stabilized. here, the state equation without the extended state variable $z_{\text {en }}$ is shown in Eq.(20).

$$
\dot{x}=A x+B u+F d
$$

The vibration mode is transformed by following Eq.(21).

$$
x=T z
$$

The state equation with departed mode is expressed in Eq.(22).

$$
\dot{z}=\tilde{A} z+\tilde{B} u+\tilde{\boldsymbol{F}} d
$$

Where, $\tilde{A}, \tilde{B}$ is defined as follows:

$$
\begin{gathered}
\tilde{\boldsymbol{A}}=\left[\begin{array}{cc}
\lambda_{1} & 0 \\
0 & \lambda_{2}
\end{array}\right] \quad \tilde{\boldsymbol{B}}=\left[\begin{array}{c}
-I_{p} /\left(\lambda_{2}-\lambda_{1}\right) \\
I_{p} /\left(\lambda_{2}-\lambda_{1}\right)
\end{array}\right] \\
\lambda_{1,2}=\frac{-C_{p} / I_{p} \pm \sqrt{\left(C_{p} / I_{p}\right)^{2}-4 K_{p} / I_{p}}}{2}
\end{gathered}
$$

The control input $u_{p}$ of the sliding mode control is the same with the one of the sliding mode control of servo system. It is also composed of linear input and nonlinear input. $u_{p}$ is expressed by 


$$
u_{p}=u l p+u_{n l p}=-(S \tilde{B})^{-1}(S \tilde{A} z)-S(S \tilde{B})^{-1} \frac{\sigma}{|\sigma|+\eta}
$$

The inputs in the $z$ direction $u_{z}$ and the direction of roll angle $u_{r}$ were calculated in the same way with the calculation procedure of $u_{p}$. And, the transforms from the virtual suspension model with the active inputs $u_{z}, u_{p}$, and $u_{r}$ to the motor torques of the support legs and the swing legs were performed as stated in section 4.3.

\subsection{About How to Deal with the Suspension and Posture Control}

In the above two type sliding mode control based on the virtual suspension model, we can design the virtual suspension to cut the low frequency vibration $(3 \mathrm{~Hz}-8 \mathrm{~Hz})$ from the walking pattern. And because the nonlinear input $u_{n l}$ of sliding mode control can supply the strong force to support the heavy weight of the robot, the trade-off problem in the design of suspension can be solved, and then the good suspension effect can be realized. On the other hand, because the design of sliding mode control is satisfied the matching condition, although the dynamic changes and the disturbances exist, the stationary errors of position and velocity can be eliminated. If we change the state variable $x_{e}=\left[\begin{array}{lll}z_{e n} & \theta_{p} & \dot{\theta}_{p}\end{array}\right]^{T}$ in Eq.(16) to $x_{e}=\left[\begin{array}{lll}z_{e n} & \theta_{p}-\theta_{p r e f} & \dot{\theta}_{p}-\dot{\theta}_{p}\end{array}\right]^{T}$, and change the state variable $x=\left[\begin{array}{ll}\theta_{p} & \dot{\theta}_{p}\end{array}\right]^{T}$ in Eq.(20) to $x=\left[\theta_{p}-\theta_{\text {pref }} \dot{\theta}_{p}-\dot{\theta}_{\text {pref }}\right]^{T}$, we can realize the posture control for the pitch angle. Here, $\theta_{\text {pref }}$ is the reference for the pitch angle. And with the same method, we can realize the posture control for the roll angle and vertical direction.

\section{Experiment and Discussion}

Both of the sliding mode control of one-type servo system and the sliding mode control based on the vibration mode coordinate were applied to the developed robot.

\subsection{Preparations for the Experiment}

Because the purpose of this study is to restrain the vibration in the $\mathrm{z}$ direction and the directions of the pitch angle and the roll angle of the robot body when the robot walks, it is necessary to obtain the outputs in these three directions. As to the output angles in the directions of the pitch angle and the roll angle, they were measured by a slant sensor. In the vertical direction, the output can be calculated from the size of the robot body and the forces in the vertical direction of each leg. Here, in order to save the cost, we don't use the force sensor to observe the forces of each leg, rather then use the motor pseudo-torque.

\subsubsection{The Observation by Using the Motor Pseudo-Torque}

The motor torque is obtained by multiplying a torque coefficient to the motor electric current. However, because the vibration caused by noise is too big, instead of the motor torque, a pseudo-torque is used as the input torque. The pseudo-torque is the calculated torque of one sampling time before. In the servo electric circuit, the calculated pseudotorque approximates to the actual consumed torque. Therefore, using the pseudo-torque, there is no the influence by the noise. Of course, a delay of one sampling time arises 
simultaneously. The influence caused by the delay can be ignored if the sampling time is small enough.

\subsubsection{Conversion from Motor Torque to Force of the Tip of Each Leg}

The force of the tip of leg, $f=\left[\begin{array}{lll}f_{x} & f_{y} & f_{z}\end{array}\right]^{T}$, was calculated from the size of each link and the inverse of Jacobi matrix. The force of the tip of leg can be obtained as expressed as follows.

$$
\begin{gathered}
f=\left(\boldsymbol{J}^{T}\right)^{-1} \boldsymbol{\tau} \\
f_{a s i s}=\tau_{2}+\frac{l_{2} \sin \theta_{2}+l_{3} \cos \left(\theta_{2}+\theta_{3}\right) \tau_{3}}{l_{3} \cos \left(\theta_{2}+\theta_{3}\right)} \\
f_{z}=\frac{1}{l_{2} \tan \left(\theta_{2}+\theta_{3}\right) \sin \theta_{2}+\cos \theta_{2}} f_{\text {asis }} \\
f_{y}=\frac{1}{\tan \theta_{1}}\left(\frac{\tau_{2}}{l_{2} \cos \theta_{2}+l_{3} \sin \left(\theta_{2}+\theta_{3}\right)}\right)+\frac{\tau_{2}}{l_{3} \cos \left(\theta_{2}+\theta_{3}\right)}-\tan \left(\theta_{2}+\theta_{3}\right) f_{z} \\
f_{x}=-\frac{\tau_{1}}{\sin \theta_{1}}\left(\frac{1}{l_{2} \cos \theta_{2}+l_{3} \sin \left(\theta_{2}+\theta_{3}\right)}\right)+\frac{1}{\tan \theta_{1}} f_{y}
\end{gathered}
$$

\subsection{Experimental Results}

The experiments were performed by three kinds of gaits. The first is with one swing leg and five support legs; the second is with two swing legs and four support legs; the third is with three swing legs and three support legs. Here, the experimental result of the first kind of gait is introduced.

\subsubsection{In the Case by Using the Sliding Mode Control of Servo System}

The experimental results in the direction of the pitch angle, the direction of the roll angle, and the vertical direction are shown in Fig.7, Fig.8 and Fig.9, respectively. They are the changes during two periods of the gait when the robot walks on the flat ground. In Fig.7, Fig. 8 and Fig.9, the thick solid line shows the response with sliding mode control on the basis of the virtual dynamic model, while the thick dashed line shows the responses with the virtual suspension model only, and the thin dotted line shows the responses without suspension for body, respectively. In Fig.7, the change of the pitch angle with the sliding mode control on the basis of the virtual dynamic model is almost zero except for the switching instance between the swing leg and the support leg, and is the best result compared to the other two control methods. The change of the roll angle in Fig. 8 gives the similar results. The efficiency of eliminating tiny vibrations in the posture of a robot body, using the robust characteristics of the sliding mode control has been verified. Furthermore, from Fig.9, it is clarified that the stationary position error of the robot's centre of gravity is almost zero when performing the sliding mode control on the basis of the virtual dynamic model. According to the experimental results, the conclusion here is that the sliding mode control based on a virtual suspension model for the control of the posture and vibration of the six-legged walking robot is effective. 
Posture and Vibration Control Based on Virtual Suspension Model for

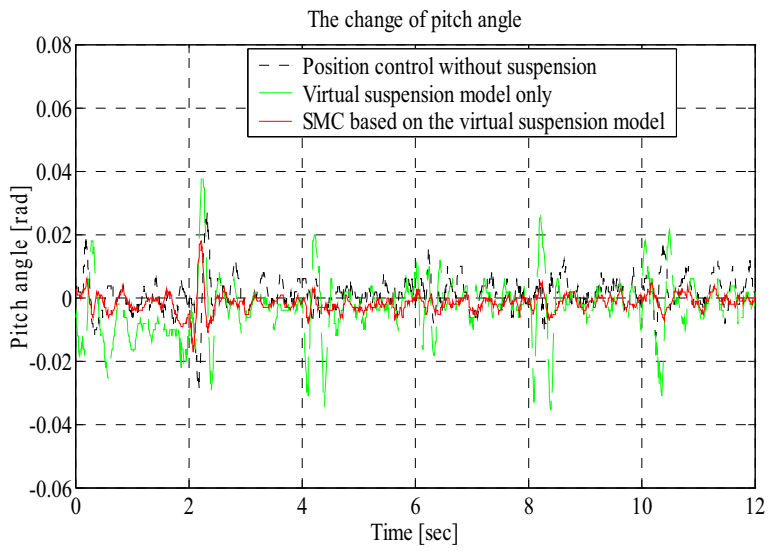

Figure 7. Changes in the direction of pitch angle (Huang, Q. et al, 2007)

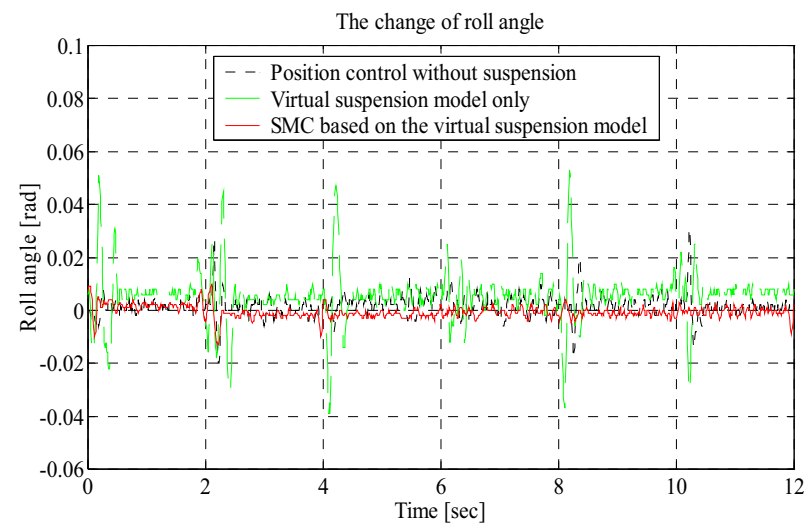

Figure 8. Changes in the direction of roll angle (Huang, Q. et al, 2007)

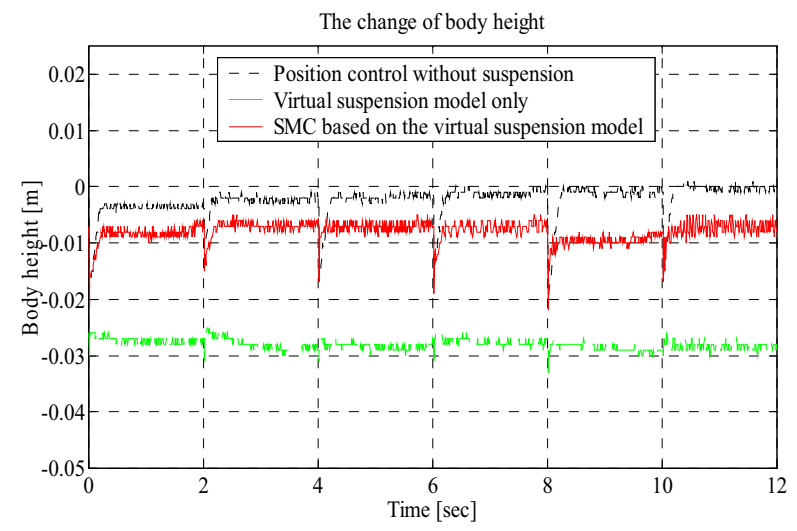

Figure 9. Changes in the vertical direction (Huang, Q. et al, 2007) 
According to the phase plane shown in Fig.14, the state variable of the pitch angle were constrained to the stable status by the control input, but after $6 \mathrm{~s}$ shown in Fig.10 and Fig.12, the control input hasn't switching status and the switch function has a trend away from zero, and this means it is difficult to arrive at the sliding mode for the state variable in this case. This reason is that the disturbance for the pitch angle is too large to satisfy a matching condition for the sliding mode control of one-type servo system.

\subsubsection{In the Case by Using Sliding Mode Control Based on the Vibration Mode Coordinate}

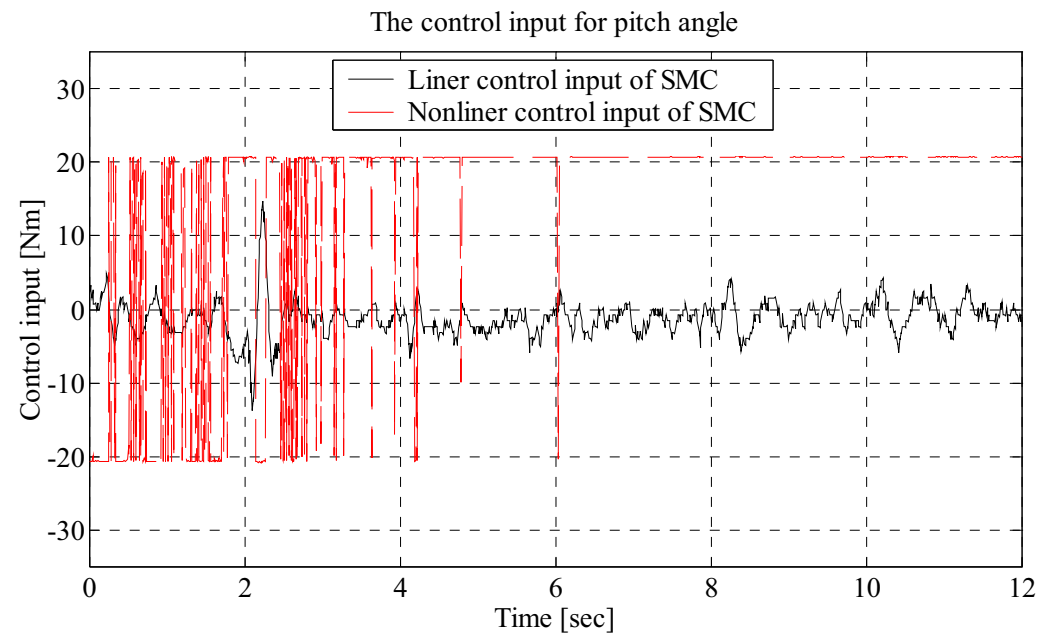

Figure 10. Control input of SMC of servo style (Huang, Q. et al, 2007)

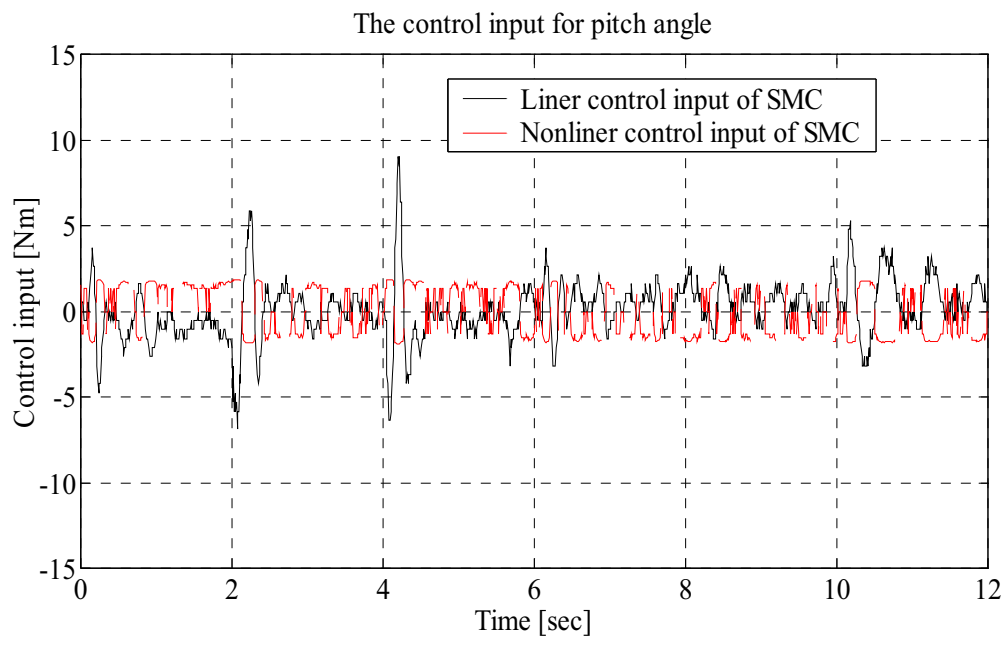

Figure 11. Control input of SMC based on mode coordinate (Huang, Q. et al, 2007) 
Posture and Vibration Control Based on Virtual Suspension Model for

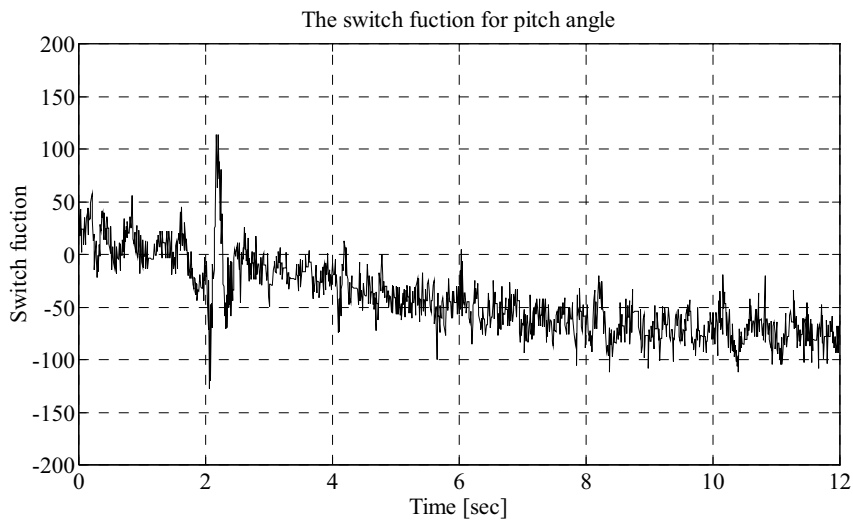

Figure 12. Switching function of SMC of servo style (Huang, Q. et al, 2007)

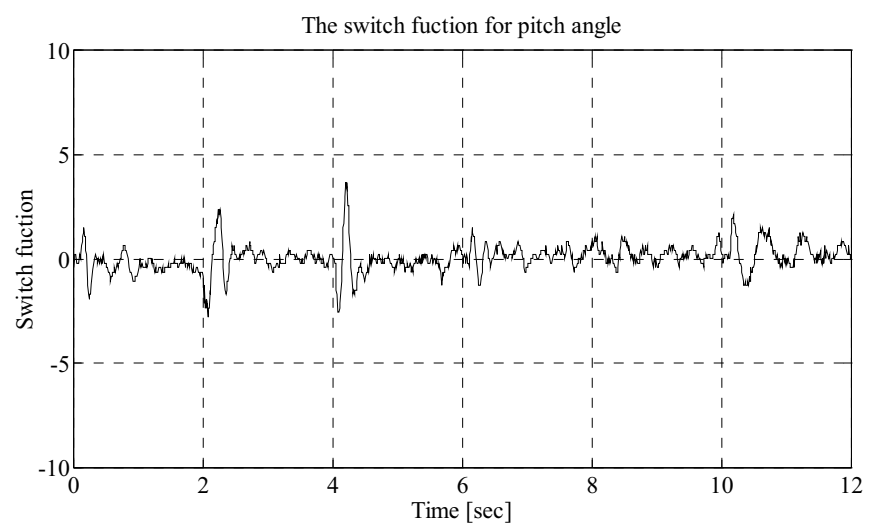

Figure 13. Switching function of SMC based on mode coordinate (Huang, Q. et al, 2007)

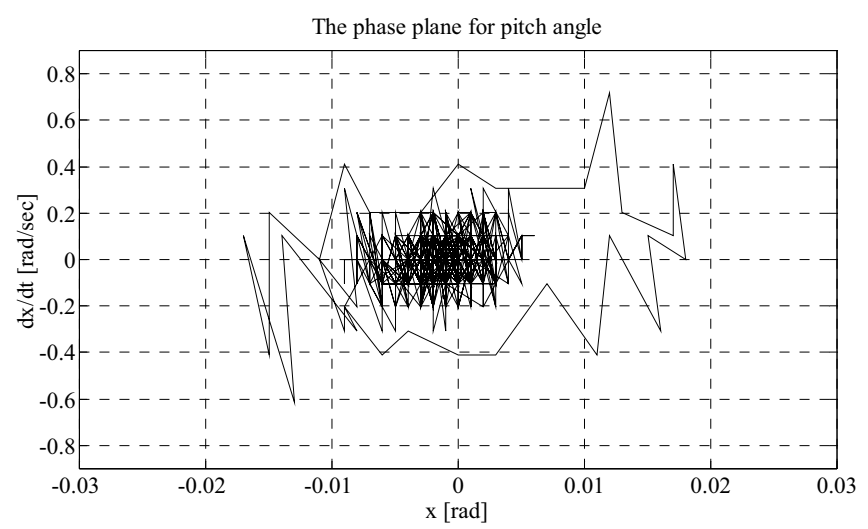

Figure 14. Phase plane of SMC of servo style (Huang, Q. et al, 2007) 


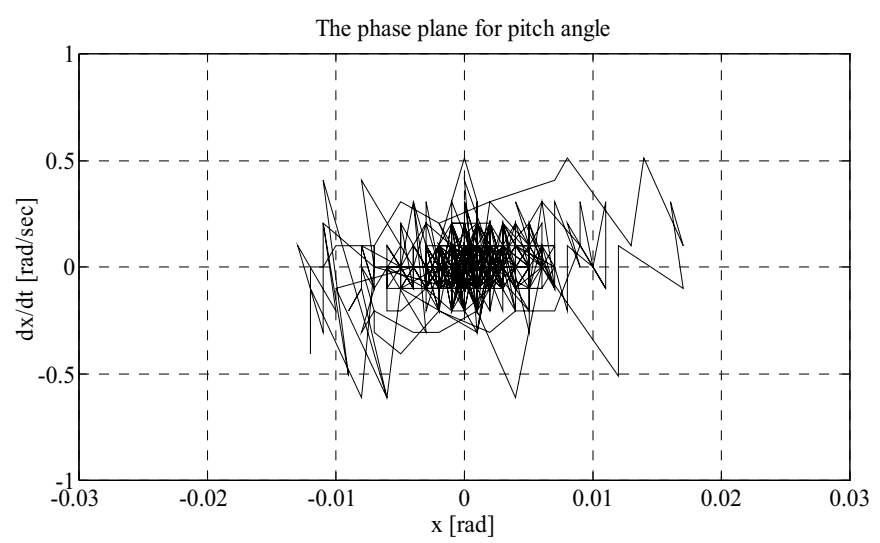

Figure 15. Phase plane of SMC based on mode coordinate (Huang, Q. et al, 2007)

The experimental results in the vertical direction, the direction of the pitch angle of the robot body, and the direction of the roll angle of the robot body, are the almost same as the results of the sliding mode control of the servo system. The control input, the switching function, and the phase plane are shown in Figures 11-15. Comparing Fig.11 with Fig.15, it is clear that in the case of the sliding mode control based on the mode coordination, the nonlinear input repeats the reconversion of the position and the negative. It shows that the system is under the sliding mode control. And comparing Fig.13 with Fig.12, it is shown that by the sliding mode control, the switching function is stable near the target value around the 0 as the centre. According to the above, it is verified that although both of the two sliding mode controls are effectively for six-legged walking robot, in the expression of the characteristic of the sliding mode control, the sliding mode control based on the mode coordination is superior to that of servo system.

\subsubsection{The Results on the Trade-off Problem in the Design of Suspension}

Firstly, we performed walking experiment by only using virtual suspension mechanism.

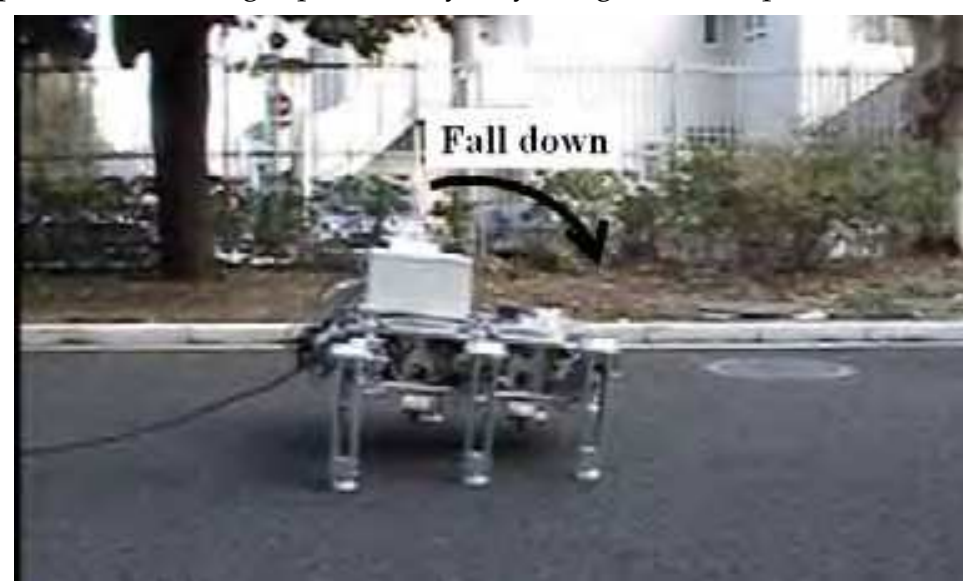

Figure 16. The broken down posture (Huang, Q. et al, 2007) 
In this case, the stiffness of suspension is slightly weak. The robot can walk, but because of the weak support force for body, the vibration exists at the instant of the foot touching the ground. The experimental results were shown as the thick dashed line in Fig.7, Fig.8and Fig.9.

And then, we increased the stiffness of the virtual suspension. In this case, the tiny vibration at the instant of the foot touching the ground was decreased, but the virtual suspension can not cut the disturbance from the walking pattern, the posture of robot body was broken down greatly at the instance that the rear swing leg was lifted as shown in the Fig.16

Next, we performed the walking experiment by using SMC based on the virtual suspension mechanism. The stable walking was realized. And then, the stable walking of the tripod gait was also realized shown as in Fig.17.

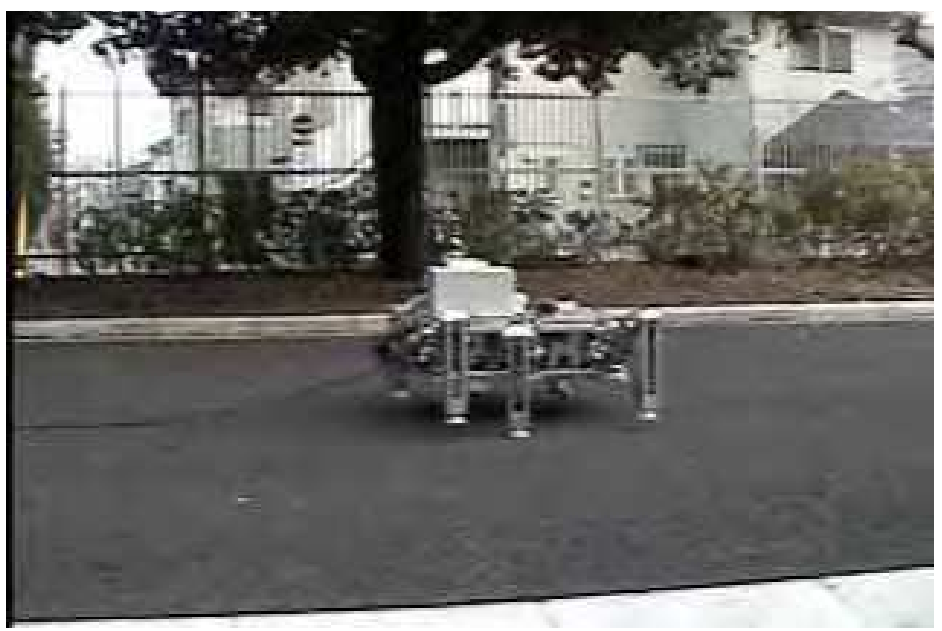

Figure 17. Tripod gait walking (Huang, Q. et al, 2007)

\section{Conclusion}

In this chapter, we treat a six-legged walking robot as a study example of the multi-legged walking robot, and introduce the newest study on a control for the posture and vibration of the robot using suspension mechanism to realize the better stability and the better adaptability of its walking for unknown rough terrain. Firstly, in order to constrain the body posture of multi-legged walking robot when it is walking, a suspension dynamic model with virtual springs and virtual dampers was constructed for the vertical direction, the directions of the pitch angle, and the direction of the roll angle of its body, respectively. And then considering the nonlinear disturbances and trade-off problem in the design of suspension, a robust control using sliding mode control based on the constructed virtual suspension model for the posture and vibration of the multi-legged walking robot was proposed. According to the above, a posture and vibration control which can keep the posture stable and decrease the vibrations in the body was realized. Furthermore, in order to use the sliding mode control effectively, two kinds of sliding mode control, the one of servo style and the one based on mode coordinate are designed. Finally, by the walking experimental results using the developed robot, we showed the efficiency of the sliding mode control based on the virtual suspension dynamic model, especially solved the trade- 
off problem of the design of suspension. Additional, by the introduction of developing a sixlegged walking robot for this study based on stable theory of wave gaits and CAD dynamic model, we offered a more efficiency developing technique for a large scale multi-DOF dynamic system, such as multi-legged walking robot. The results of this study for the above six-legged robot can be applicable to the other multi-legged walking robots.

In the near future, we will extend the posture and vibration control from the abovementioned 3-DOF (pitch, roll, $\mathrm{z}$ axis) up to 6-DOF in consideration of forward (y axis), side ( $x$ axis) and rotation (yaw). And then, we will design a hierarchical control system for multilegged walking robot, which is combined the above-introduced posture and vibration control for the body with a position and force hybrid control for the legs, to realize the stable walking on unknown rough terrain and over striding obstacles.

\section{References}

Shin-Min Song \& Keneth J.Waldron (1989). Machines that walk, the adaptive suspension vehicle, The MIT Press Cambridge, Massachusetts London, England

Kan Yoneda, Hiroyuki Iiyama, Shigeo Hirose (1994). Sky-Hook Suspension Control of a Quadruped Walking Vehicle, Journal of the Robotics Society of Japan, Vol.12, No.7, 1066-1071 (In Japanese)

Qingjiu Huang, Kenzo Nonami, etc. (2000). CAD Model Based Autonomous Locomotion of Quadruped Robot by Using Correction of Trajectory Planning with RNN, Special Issue on Frontiers of Motion and Vibration Control, JSME International Journal, Series C, Vol.43, No.3, pp.653-663

Qingjiu Huang \& Kenzo Nonami (2002). Neuro-Based Position and Force Hybrid Control of Six-Legged Walking Robot, Special Issue on Modern Trends on Mobile Robotics, Journal of Robotics and Mechatronics, Vol.14, No.4, pp.534-543

Qingjiu Huang \& Kenzo Nonami (2003). Humanitarian Mine Detecting Six-Legged Walking Robot and Hybrid Neuro Walking Control with Position/Force Control, Special Issue on Computational Intelligence in Mechatronic Systems, Mechatronics, Vol.13, No.89, pp.773-790

Nurkan Yagiz, Ismail Yuksek, Selim Sivriogle (2000). Robust Control of Active Suspension for a full Vehicle Model Using Sliding Mode Control, JSME International Journal, Series C, Vol.43, No.2, pp.253-258

Makoto Yokoyama, J.K. Hedrick, Shigehiro Toyama (2001). A Sliding Mode Controller for Semi-Active Suspension System, Transactions of the Japan Society of Mechanical Engineers, Series C, Vol.67, No.657, pp.1449-1454 (in Japanese)

Qingjiu Huang, Masayoshi Yanai, Kyosuke Oon, Kenzo Nonami (2004). Robust Control of Posture and Vibration Based on Virtual Suspension Model for Six-Legged Walking Robot, Proceedings of the 7th international conference of Motion and Vibration Control, Washington University in St. Louis, America, CD-ROM, No.41

Kenzo Nonami \& Hongqi Tian (1994). Sliding Mode Control, CORONA Publishing Co., Ltd. (In Japanese)

Qingjiu Huang, Yasuyuki Fukuhara, Xuedong Chen (2007). Posture and Vibration Control Based on Virtual Suspension Model Using Sliding Mode Control for Six-Legged Walking Robot, Special Issue on New Trends of Motion and Vibration Control, Journal of System Design and Dynamics of JSME, Vol.1, No.2, pp.180-191

LMS DADS (Dynamics Analysis and Design System) is a product of LMS International. 


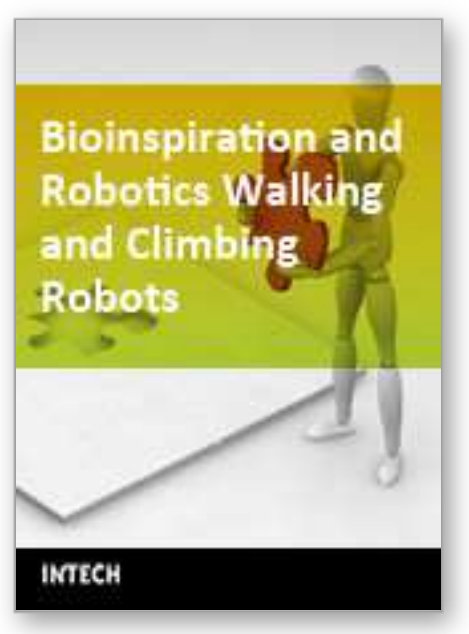

\section{Bioinspiration and Robotics Walking and Climbing Robots \\ Edited by Maki K. Habib}

ISBN 978-3-902613-15-8

Hard cover, 544 pages

Publisher I-Tech Education and Publishing

Published online 01, September, 2007

Published in print edition September, 2007

Nature has always been a source of inspiration and ideas for the robotics community. New solutions and technologies are required and hence this book is coming out to address and deal with the main challenges facing walking and climbing robots, and contributes with innovative solutions, designs, technologies and techniques. This book reports on the state of the art research and development findings and results. The content of the book has been structured into 5 technical research sections with total of 30 chapters written by well recognized researchers worldwide.

\section{How to reference}

In order to correctly reference this scholarly work, feel free to copy and paste the following:

Qingjiu Huang (2007). Posture and Vibration Control Based on Virtual Suspension Model for Multi-Legged Walking Robot, Bioinspiration and Robotics Walking and Climbing Robots, Maki K. Habib (Ed.), ISBN: 978-3902613-15-8, InTech, Available from:

http://www.intechopen.com/books/bioinspiration_and_robotics_walking_and_climbing_robots/posture_and_vib ration_control_based_on_virtual_suspension_model_for_multi-legged_walking_robot

\section{INTECH}

open science | open minds

\author{
InTech Europe \\ University Campus STeP Ri \\ Slavka Krautzeka 83/A \\ 51000 Rijeka, Croatia \\ Phone: +385 (51) 770447 \\ Fax: +385 (51) 686166 \\ www.intechopen.com
}

\author{
InTech China \\ Unit 405, Office Block, Hotel Equatorial Shanghai \\ No.65, Yan An Road (West), Shanghai, 200040, China \\ 中国上海市延安西路65号上海国际贵都大饭店办公楼 405 单元 \\ Phone: +86-21-62489820 \\ Fax: +86-21-62489821
}


(C) 2007 The Author(s). Licensee IntechOpen. This chapter is distributed under the terms of the Creative Commons Attribution-NonCommercial-ShareAlike-3.0 License, which permits use, distribution and reproduction for non-commercial purposes, provided the original is properly cited and derivative works building on this content are distributed under the same license. 